\title{
Local boundedness of quasi-minimizers of integral functionals with variable exponent anisotropic growth and applications
}

Xianling Fan

\begin{abstract}
The local boundedness of local quasi-minimizers of integral functionals with variable exponent anisotropic $\vec{p}(x)$ growth under suitable assumptions is proved. Based on this result, the global boundedness and the Lipschitz continuity of weak solutions of Dirichlet or Neumann boundary value problems for the $\vec{p}(x)$-Laplace type equations are obtained.
\end{abstract}

Mathematics Subject Classification (2000). 49N60, 35J70.

Keywords. Anisotropic, Variable exponent, Quasi-minimizer, Boundedness, Weak solution.

\section{Introduction}

Variational problems and differential equations with $(p, q)$ non-standard growth have been a very active field of investigation in recent years. We refer to the overview paper [26] for the advances of this field. The anisotropic $\vec{p}$ growth, where $\vec{p}=\left(p_{1}, p_{2}, \ldots, p_{N}\right)$, and the variable exponent $p(x)$ growth are two important special cases of $(p, q)$ growth. Recently, some authors (see $[3,27,35-37])$ investigated the existence of weak solutions of elliptic and parabolic equations with variable exponent anisotropic growth, where the $\vec{p}(x)$ Laplacian

$$
-\sum_{i=1}^{N} D_{i}\left(\left|D_{i} u\right|^{p_{i}(x)-2} D_{i} u\right)
$$

is involved. This is a new and interesting research topic. The $\vec{p}(x)$ growth, i.e. the variable exponent anisotropic growth, is a generalization of the $\vec{p}$ growth and the $p(x)$ growth, and itself is also a special case of $(p, q)$ growth. 
The regularity for variational problems and differential equations with nonstandard growth has been studied extensively and many interesting results have been obtained, for example, we refer to $[8,12,34]$ for general $(p, q)$ growth case, refer to $[1,4-6,19-21,30-33]$ for $\vec{p}$ growth case and refer to $[2,9,10,13-$ $17,25,41,44,45]$ for $p(x)$ growth case. To our knowledge, the regularity for the $\vec{p}(x)$ growth case has not yet been studied specially and systematically.

In the study of the regularity of weak solutions, the local boundedness of weak solutions occupies an especially important place. More recently, Cupini et al. [7] obtained the local boundedness of local minimizers of the integral functionals of the form

$$
\mathcal{F}(u)=\int_{\Omega} f\left(x,\left|D_{1} u\right|, \ldots,\left|D_{N} u\right|\right) \mathrm{d} x
$$

under the assumptions that the function $f(x, \xi)$ is convex and of class $C^{1}$ with respect to $\xi$, and $f$ satisfies $\left(p_{i}, q\right)$ growth condition with $q<\bar{p}^{*}$, where $\bar{p}$ is the harmonic average for $\vec{p}$, that is

$$
\frac{1}{\bar{p}}=\frac{1}{N} \sum_{i=1}^{N} \frac{1}{p_{i}}
$$

and

$$
\bar{p}^{*}=\left\{\begin{array}{ll}
\frac{N \bar{p}}{N-\bar{p}} & \text { if } \bar{p}<N \\
+\infty & \text { if } \bar{p} \geq N
\end{array} .\right.
$$

This is a very nice and useful result. The $\left(p_{i}, q\right)$ growth includes the $\vec{p}(x)$ growth as a special case, and the functional $\mathcal{F}(u)$ includes the functional $\int_{\Omega} \sum_{i=1}^{N}\left|D_{i} u\right|^{p_{i}(x)} \mathrm{d} x$ as a special case.

The $\vec{p}(x)$ growth, as a special case of the $\left(p_{i}, q\right)$ growth, has some selfderived features distinguishing the general $\left(p_{i}, q\right)$ growth. A main aim of the present paper is to obtain the local boundedness of the quasi-minimizers of integral functionals with $\vec{p}(x)$ growth under weaker assumptions than those used in [7]. A main result of the present paper is the following theorem in which the following notations are used:

$$
\begin{aligned}
C_{+}^{0}(\bar{\Omega}) & =\left\{q \in C^{0}(\bar{\Omega}): q(x) \geq 1 \text { for } x \in \bar{\Omega}\right\}, \\
\vec{p}(x) & =\left(p_{1}(x), p_{2}(x), \ldots, p_{N}(x)\right), \\
\frac{1}{\bar{p}(x)} & =\frac{1}{N} \sum_{i=1}^{N} \frac{1}{p_{i}(x)}, \bar{p}^{*}(x)=\left\{\begin{array}{ll}
\frac{N \bar{p}(x)}{N-\bar{p}(x)} & \text { if } \bar{p}(x)<N \\
+\infty & \text { if } \bar{p}(x) \geq N
\end{array}, \forall x \in \bar{\Omega},\right. \\
D_{i} u & =\frac{\partial u}{\partial x_{i}}, \quad D u=\left(D_{1} u, \ldots, D_{N} u\right) .
\end{aligned}
$$

Theorem 1.1. Let $\Omega \subset \mathbb{R}^{N}$ be a bounded domain and $\vec{p}(\cdot) \in\left(C_{+}^{0}(\bar{\Omega})\right)^{N}$ satisfy condition

$$
p_{i}(x)<\bar{p}^{*}(x) \quad \text { for } x \in \Omega \text { and } i=1,2, \ldots, N \text {. }
$$


Let $f: \Omega \times \mathbb{R} \times \mathbb{R}^{N} \rightarrow \mathbb{R}$ be a Carathéodory function satisfying the following conditions:

$$
\begin{aligned}
& c_{0} \sum_{i=1}^{N}\left|\xi_{i}\right|^{p_{i}(x)}-c_{1}|s|^{q(x)}-c_{1} g(x) \\
& \quad \leq f(x, s, \xi) \leq c_{2} \sum_{i=1}^{N}\left|\xi_{i}\right|^{p_{i}(x)}+c_{2}|s|^{q(x)}+c_{2} g(x),
\end{aligned}
$$

where $x \in \Omega, s \in \mathbb{R}, \xi=\left(\xi_{1}, \ldots, \xi_{N}\right) \in \mathbb{R}^{N}, c_{i}$ is a positive constant, $q \in C_{+}^{0}(\bar{\Omega})$ and

$$
q(x)<\bar{p}^{*}(x) \text { for all } x \in \Omega,
$$

$g(x) \geq 0$ for a.e. $x \in \Omega$,

$$
g \in L_{l o c}^{r(\cdot)}(\Omega) \text { with } r \in C_{+}^{0}(\bar{\Omega}), r(x)>\max \left\{1, \frac{N}{\bar{p}(x)}\right\} \text { for } x \in \Omega \text {. }
$$

Suppose that $u \in W_{l o c}^{1, \vec{p}(\cdot)}(\Omega)$ is a local quasi-minimizer of the integral functional

$$
J(u)=\int_{\Omega} f(x, u(x), D u(x)) d x
$$

over $\Omega$ in $W_{l o c}^{1, \vec{p}(\cdot)}(\Omega)$. Then $u \in L_{l o c}^{\infty}(\Omega)$.

For the definitions of $L^{r(\cdot)}(\Omega), W_{l o c}^{1, \vec{p}(\cdot)}(\Omega)$ and the local quasi-minimizers of $J$ see Sect. 2 below.

Compared with the main result of [7], in our Theorem 1.1, $f(x, s, \xi)$ can be dependent on $s$ and need not be convex and of class $C^{1}$ with respect to $\xi$. For example, the function $f(x, \xi):=\sum_{i=1}^{N}\left(2+\cos \xi_{i}\right)\left|\xi_{i}\right|^{p_{i}(x)}$ is not convex with respect to $\xi$, but it can satisfy the assumptions of Theorem 1.1. In addition, Theorem 1.1 deals with the local quasi-minimizers which include the local minimizers, the weak solutions of corresponding elliptic equations and the weak solutions of corresponding variational inequalities as the special cases. Some arguments used in [7] to prove the local boundedness of local minimizers cannot be carried out for the local quasi-minimizer case because some characteristic properties of the local minimizer are used in [7].

The present paper is organized as follows. In Sect. 2 we give some preliminaries on the variable exponent anisotropic Sobolev spaces and the quasi-minimizers. In particular, we give a compact imbedding result and a $C^{\infty}$-density result for the variable exponent anisotropic Sobolev spaces, see Theorems 2.1-2.3, which are themselves also of independent interest. In Sect. 3 we give the proof of Theorem 1.1. In Sect. 4, we give some applications of Theorem 1.1 to the regularity of weak solutions of Dirichlet (or Neumann) boundary value problems for the $\vec{p}(x)$-Laplace type equations. Using Theorem 1.1 we obtain the global boundedness of the weak solutions and furthermore, using the result of [31], we obtain the Lipschitz continuity of the weak solutions. 


\section{Preliminaries}

Let $\Omega \subset \mathbb{R}^{N}$ be a bounded domain. $S(\Omega)$ denotes the set of all measurable real-valued functions defined on $\Omega$. Define

$$
L_{+}^{\infty}(\Omega)=\left\{u \in L^{\infty}(\Omega): u(x) \geq 1 \text { for a.e. } x \in \Omega\right\} .
$$

For $p \in L_{+}^{\infty}(\Omega)$, the variable exponent Lebesgue space $L^{p(\cdot)}(\Omega)$ is defined by

$$
L^{p(\cdot)}(\Omega)=\left\{u \in S(\Omega): \int_{\Omega}|u(x)|^{p(x)} \mathrm{d} x<\infty\right\}
$$

with the norm

$$
\|u\|_{L^{p(\cdot)}(\Omega)}=\|u\|_{p(\cdot)}=\inf \left\{\lambda>0: \int_{\Omega}\left|\frac{u(x)}{\lambda}\right|^{p(x)} \mathrm{d} x \leq 1\right\} .
$$

Let $\vec{p}(\cdot) \in\left(L_{+}^{\infty}(\Omega)\right)^{N}$. Define

$$
W^{1, \vec{p}(\cdot)}(\Omega)=\left\{u \in W^{1,1}(\Omega): D_{i} u \in L^{p_{i}(\cdot)}(\Omega) \text { for } i=1,2, \ldots, N\right\}
$$

with the norm $\|u\|_{W^{1, \vec{p}(\cdot)(\Omega)}}=\|u\|_{L^{1}(\Omega)}+\sum_{i=1}^{N}\left\|D_{i} u\right\|_{L^{p_{i}(\cdot)}(\Omega)}$.

We will also use the space $W_{0}^{1,1}(\Omega) \cap W^{1, \vec{p}(\cdot)}(\Omega)$, a subspace of $W^{1, \vec{p}(\cdot)}$ $(\Omega)$, in it the norm $\|u\|_{W^{1, \vec{p}(\cdot)(\Omega)}}$ is installed.

The variable exponent anisotropic Sobolev space $W^{1, \vec{p}(\cdot)}(\Omega)$ is a generalization of the constant exponent anisotropic Sobolev space $W^{1, \vec{p}}(\Omega)$ and the variable exponent isotropic Sobolev space $W^{1, p(\cdot)}(\Omega)$. We refer to $[1,19$, $29,38,39,42,43]$ for basic results on $W^{1, \vec{p}}(\Omega)$ and refer to $[9,11,18,28,40,41]$ for basic results on $W^{1, p(\cdot)}(\Omega)$.

For the Banach spaces $X$ and $Y$, the notation $X \hookrightarrow Y$ denotes that $X$ is continuously imbedded into $Y$, and the notation $X \hookrightarrow \hookrightarrow Y$ denotes that $X$ is compactly imbedded into $Y$.

It is well known that, in the constant exponent case, when $\vec{p}=$ $\left(p_{1}, p_{2}, \cdots, p_{N}\right)$ and $\bar{p}<N$, in general, the imbedding $W^{1, \vec{p}}(\Omega) \hookrightarrow L^{\bar{p}^{*}}(\Omega)$ may be not true even if $\Omega$ is a cube (see for example [29]). Acerbi and Fusco [1] established the following imbedding theorem.

Proposition 2.1. (1) Let $\Omega \subset \mathbb{R}^{N}$ be a rectangular domain with edges parallel to the coordinate axes and $\vec{p}=\left(p_{1}, p_{2}, \cdots, p_{N}\right)$ with $p_{i} \geq 1$ and

$$
p_{i}<\bar{p}^{*} \quad \text { for } i=1,2, \ldots, N \text {. }
$$

Then $W^{1, \vec{p}}(\Omega) \hookrightarrow L^{\bar{p}^{*}}(\Omega)$ if $\bar{p}<N$, and $W^{1, \vec{p}}(\Omega) \hookrightarrow L^{q}(\Omega)$ for any $q \in[1, \infty)$ if $\bar{p} \geq N$.

(2) If use $W_{0}^{1,1}(\Omega) \cap W^{1, \vec{p}}(\Omega)$ instead of $W^{1, \vec{p}}(\Omega)$ in statement 1$)$, then the corresponding imbedding results for $W_{0}^{1,1}(\Omega) \cap W^{1, \vec{p}}(\Omega)$ are true for the generic bounded open set $\Omega$, not only for a rectangular domain.

Noting that $W^{1, \vec{p}}(\Omega) \hookrightarrow \hookrightarrow L^{1}(\Omega)$, from Proposition 2.1 we have the following 
Corollary 2.1. Under the assumptions of Proposition 2.1, the compact imbedding $W^{1, \vec{p}}(\Omega) \hookrightarrow \hookrightarrow L^{q}(\Omega)$ (respectively, $W_{0}^{1,1}(\Omega) \cap W^{1, \vec{p}}(\Omega) \hookrightarrow \hookrightarrow L^{q}(\Omega)$ ) holds for $1 \leq q<\bar{p}^{*}$.

Note that in Proposition 2.1, condition (2.1) is key. In many examples on the non-regularity for $\vec{p}$ growth case, condition (2.1) is not satisfied.

In what follows, for brevity, a rectangular domain (or a cube) with edges parallel to the coordinate axes is simply said to be a rectangular domain (or a cube). For $x \in \mathbb{R}^{N}$ and $\varepsilon>0$, we denote by $K(x, \varepsilon)$ the $N$-dimensional open cube with center $x$ and edge length $\varepsilon$. For $E \subset \Omega$, the notation $E \subset \subset \Omega$ means that $\bar{E}$ is compact and $\bar{E} \subset \Omega$. For $q \in L_{+}^{\infty}(\Omega)$ and $E \subset \Omega$, define

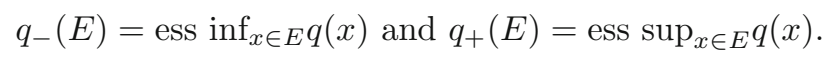

For the spaces $W^{1, \vec{p}(\cdot)}(\Omega)$ and $W_{0}^{1,1}(\Omega) \cap W^{1, \vec{p}(\cdot)}(\Omega)$, we give the following compact imbedding theorem which is an extension of Corollary 2.1 to the variable exponent case and is a basis of proving Theorem 1.1.

Theorem 2.1. (1) Let $\Omega \subset \mathbb{R}^{N}$ be a rectangular domain and $\vec{p}(\cdot) \in\left(C_{+}^{0}(\bar{\Omega})\right)^{N}$ satisfy

$$
p_{i}(x)<\bar{p}^{*}(x) \text { for } x \in \bar{\Omega} \text { and } i=1,2, \ldots, N .
$$

Suppose that $q \in C_{+}^{0}(\bar{\Omega})$ satisfies condition

$$
q(x)<\bar{p}^{*}(x) \text { for } x \in \bar{\Omega} \text { and } i=1,2, \ldots, N .
$$

Then $W^{1, \vec{p}(\cdot)}(\Omega) \hookrightarrow \hookrightarrow L^{q(\cdot)}(\Omega)$.

(2) If use $W_{0}^{1,1}(\Omega) \cap W^{1, \vec{p}(\cdot)}(\Omega)$ instead of $W^{1, \vec{p}(\cdot)}(\Omega)$ in statement 1$)$, then the corresponding imbedding result is true for the generic bounded open set $\Omega$, not only for a rectangular domain.

Proof. (1) Let any $x_{0} \in \bar{\Omega}$ be given. By (2.2), (2.3) and the continuity of $\vec{p}(\cdot)$ and $q(\cdot)$ on $\bar{\Omega}$, there exists an open cube $K\left(x_{0}, \varepsilon_{x_{0}}\right)$ such that, if denote $G=K\left(x_{0}, \varepsilon_{x_{0}}\right) \cap \Omega$ and $\overrightarrow{p_{-}(G)}=\left(p_{1,-}(G), p_{2,-}(G), \ldots, p_{N,-}(G)\right)$, then

$$
p_{i,-}(G)<{\overline{p_{-}(G)}}^{*} \text { for } i=1,2, \ldots, N \text {, and } q_{+}(G)<{\overline{p_{-}(G)}}^{*},
$$

where $\overline{p_{-}(G)}$ is the harmonic average for $\overrightarrow{p_{-}(G)}$. By Corollary $2.1, W^{1, \overrightarrow{p_{-}(G)}}$ $(G) \hookrightarrow \hookrightarrow L^{q_{+}(G)}(G)$. It is obvious that

$$
W^{1, \vec{p}(\cdot)}(G) \hookrightarrow W^{1, \overrightarrow{p_{-}(G)}}(G) \quad \text { and } \quad L^{q_{+}(G)}(G) \hookrightarrow L^{q(\cdot)}(G),
$$

thus we have that $W^{1, \vec{p}(\cdot)}(G) \hookrightarrow \hookrightarrow L^{q(\cdot)}(G)$. From this, using the finite covering theorem for the compact set $\bar{\Omega}$, we can obtain that $W^{1, \vec{p}(\cdot)}(\Omega) \hookrightarrow \hookrightarrow$ $L^{q(\cdot)}(\Omega)$.

(2) Let $\Omega \subset \mathbb{R}^{N}$ be a bounded open set and let any $x_{0} \in \bar{\Omega}$ be given. Let $K\left(x_{0}, \varepsilon_{x_{0}}\right)$ and $G=K\left(x_{0}, \varepsilon_{x_{0}}\right) \cap \Omega$ be as in the proof of statement (1). In the case when $x_{0} \in \Omega$, taking the cube $K\left(x_{0}, \varepsilon_{x_{0}}\right)$ small enough such that $K\left(x_{0}, \varepsilon_{x_{0}}\right) \subset \Omega$, then $G=K\left(x_{0}, \varepsilon_{x_{0}}\right)$ is a cube and thus, as is proved in (1) above, $W^{1, \vec{p}(\cdot)}(G) \hookrightarrow \hookrightarrow L^{q(\cdot)}(G)$. In the case when $x_{0} \in \partial \Omega$, for any $u \in W_{0}^{1,1}(\Omega) \cap W^{1, \vec{p}(\cdot)}(\Omega)$, defining additionally $u(x)=0$ for $x \in K\left(x_{0}, \varepsilon_{x_{0}}\right) \backslash \Omega$, 
then $u \in W^{1, \overrightarrow{p_{-}(G)}}\left(K\left(x_{0}, \varepsilon_{x_{0}}\right)\right)$. By Corollary 2.1, $W^{1, \overrightarrow{p_{-}(G)}}\left(K\left(x_{0}, \varepsilon_{x_{0}}\right)\right) \hookrightarrow \hookrightarrow$ $L^{q_{+}(G)}\left(K\left(x_{0}, \varepsilon_{x_{0}}\right)\right)$. From this and the compactness of $\bar{\Omega}$ we can see that statement (2) is true.

Noting that in Theorem 2.1, (2.2) holds, we immediately have the following

Corollary 2.2. Under the assumptions of Theorem 2.1, $W^{1, \vec{p}(\cdot)}(\Omega) \hookrightarrow \hookrightarrow$ $L^{p_{i}(\cdot)}(\Omega)$ (respectively, $W_{0}^{1,1}(\Omega) \cap W^{1, \vec{p}(\cdot)}(\Omega) \hookrightarrow \hookrightarrow L^{p_{i}(\cdot)}(\Omega)$ ) for $i=1,2, \ldots, N$.

It is well-known that, in the case that $p_{1}(\cdot)=p_{2}(\cdot)=\cdots=p_{N}(\cdot)=$ $p(\cdot)$, if $p(\cdot)$ is not log-Hölder continuous, then $C^{\infty}(\bar{\Omega})$ need not be dense in $W^{1, p(\cdot)}(\Omega)$, and $C_{0}^{\infty}(\Omega)$ need not be dense in $W_{0}^{1,1}(\Omega) \cap W^{1, p(\cdot)}(\Omega)$ (see $[9$, $13,18,41,44])$. This shows that, for a general variable exponent $\vec{p}(\cdot), C^{\infty}(\bar{\Omega})$ need not be dense in $W^{1, \vec{p}(\cdot)}(\Omega)$, and $C_{0}^{\infty}(\Omega)$ need not be dense in $W_{0}^{1,1}(\Omega) \cap$ $W^{1, \vec{p}(\cdot)}(\Omega)$. We denote by $W_{\infty}^{1, \vec{p}(\cdot)}(\Omega)$ the closure of $C^{\infty}(\bar{\Omega})$ in $W^{1, \vec{p}(\cdot)}(\Omega)$, and by $W_{0}^{1, \vec{p}(\cdot)}(\Omega)$ the closure of $C_{0}^{\infty}(\Omega)$ in $W^{1, \vec{p}(\cdot)}(\Omega)$. Then obviously $W_{\infty}^{1, \vec{p}(\cdot)}(\Omega) \subset W^{1, \vec{p}(\cdot)}(\Omega)$ and $W_{0}^{1, \vec{p}(\cdot)}(\Omega) \subset W_{0}^{1,1}(\Omega) \cap W^{1, \vec{p}(\cdot)}(\Omega)$, but in general, $W_{\infty}^{1, \vec{p}(\cdot)}(\Omega) \neq W^{1, \vec{p}(\cdot)}(\Omega)$ and $W_{0}^{1, \vec{p}(\cdot)}(\Omega) \neq W_{0}^{1,1}(\Omega) \cap W^{1, \vec{p}(\cdot)}(\Omega)$.

From Corollary 2.2 and the known $C_{0}^{\infty}(\Omega)$-density result for the variable exponent Lebesgue-Sobolev spaces (see $[9,13,18,41,44])$ we immediately have the following

Theorem 2.2. Let $\Omega \subset \mathbb{R}^{N}$ be a bounded domain with Lipschitz boundary and $\vec{p}(\cdot)$ satisfy (2.2). If for each $i=1,2, \ldots, N, p_{i}: \bar{\Omega} \rightarrow \mathbb{R}$ is log-Hölder continuous, that is, there exists a positive constant $L$ such that

$$
\left|p_{i}(x)-p_{i}(y)\right| \leq \frac{L}{-\ln |x-y|} \text { for } x, y \in \bar{\Omega} \text { with }|x-y| \leq \frac{1}{2},
$$

then $C_{0}^{\infty}(\Omega)$ is dense in $W_{0}^{1,1}(\Omega) \cap W^{1, \vec{p}(\cdot)}(\Omega)$, and thus $W_{0}^{1, \vec{p}(\cdot)}(\Omega)=$ $W_{0}^{1,1}(\Omega) \cap W^{1, \vec{p}(\cdot)}(\Omega)$.

Based on Theorem 2.2, using the reflection extension method (see for example [1]), we can obtain the following

Theorem 2.3. Let $\Omega \subset \mathbb{R}^{N}$ be a rectangular domain and $\vec{p}(\cdot) \in\left(L_{+}^{\infty}(\Omega)\right)^{N}$. If for each $i=1,2, \ldots, N, p_{i}: \bar{\Omega} \rightarrow \mathbb{R}$ is log-Hölder continuous, then $C^{\infty}(\bar{\Omega})$ is dense in $W^{1, \vec{p}(\cdot)}(\Omega)$, and thus $W_{\infty}^{1, \vec{p}(\cdot)}(\Omega)=W^{1, \vec{p}(\cdot)}(\Omega)$.

Proof. Denote by $3 \Omega$ the rectangular domain with same center as $\Omega$ and three times the side, and let $\eta$ be a $C^{\infty}$ cut-off function between $\Omega$ and $3 \Omega$. We extend the function $p_{i}$ by reflections to a function on $\overline{3 \Omega}$, denoted still by $p_{i}$. For $u \in W^{1, \vec{p}(\cdot)}(\Omega)$, we may extend it by reflections to a function $\widetilde{u} \in W^{1, \vec{p}(\cdot)}(3 \Omega)$. Set $v=\eta \widetilde{u}$. Then $v \in W_{0}^{1,1}(3 \Omega) \cap W^{1, \vec{p}(\cdot)}(3 \Omega)$. By Theorem $2.2, v$ can be approximated by the functions in $C_{0}^{\infty}(3 \Omega)$, and thus $u$ can be approximated by the functions in $C^{\infty}(\bar{\Omega})$. Theorem 2.3 is proved. 
It is clear that $\sum_{i=1}^{N}\left\|D_{i} u\right\|_{L^{p_{i}(\cdot)}(\Omega)}$ is an equivalent norm on $W_{0}^{1, \vec{p}(\cdot)}(\Omega)$ (or on $\left.W_{0}^{1,1}(\Omega) \cap W^{1, \vec{p}(\cdot)}(\Omega)\right)$.

Define

$$
\begin{aligned}
& W_{l o c}^{1, \vec{p}(\cdot)}(\Omega)=\left\{u \in S(\Omega): u \in W^{1, \vec{p}(\cdot)}(G) \text { for any open set } G \subset \subset \Omega\right\}, \\
& W_{\infty, l o c}^{1, \vec{p}(\cdot)}(\Omega)=\left\{u \in S(\Omega): u \in W_{\infty}^{1, \vec{p}(\cdot)}(G) \text { for any open set } G \subset \subset \Omega\right\} .
\end{aligned}
$$

Now let $f: \Omega \times \mathbb{R} \times \mathbb{R}^{N} \rightarrow \mathbb{R}$ be as in Theorem 1.1. For $u \in W_{\text {loc }}^{1, \vec{p}(\cdot)}(\Omega)$ and $G \subset \subset \Omega$, define

$$
J(u, G)=\int_{G} f(x, u, D u) \mathrm{d} x .
$$

Definition 2.1. A function $u \in W_{l o c}^{1, \vec{p}(\cdot)}(\Omega)$ (respectively, $u \in W_{\infty, l o c}^{1, \vec{p}(\cdot)}(\Omega)$ ) is said to be a quasi-minimizer of $J$ over $\Omega$ in $W_{l o c}^{1, \vec{p}(\cdot)}(\Omega)$ (respectively, in $\left.W_{\infty, l o c}^{1, \vec{p}(\cdot)}(\Omega)\right)$ if there exists a constant $Q \geq 1$ such that for every $v \in W_{l o c}^{1, \vec{p}(\cdot)}(\Omega)$ (respectively, $\left.v \in W_{\infty, l o c}^{1, \vec{p}(\cdot)}(\Omega)\right)$ with $E:=\operatorname{supp}(u-v) \subset \subset \Omega$ there holds

$$
J(u, E) \leq Q J(v, E) .
$$

The constant $Q$ is called a proportion coefficient of the quasi-minimizer $u$.

Definition 2.2. A function $u \in W_{l o c}^{1, \vec{p}(\cdot)}(\Omega)$ (respectively, $u \in W_{\infty, l o c}^{1, \vec{p}(\cdot)}(\Omega)$ ) is said to be a local quasi-minimizer of $J$ over $\Omega$ in $W_{l o c}^{1, \vec{p}(\cdot)}(\Omega)$ (respectively, in $\left.W_{\infty, l o c}^{1, \vec{p}(\cdot)}(\Omega)\right)$ if for every $x \in \Omega$, there exists an open neighborhood $G \subset \Omega$ of $x$ such that $u$ is a quasi-minimizer of $J$ over $G$ in $W_{l o c}^{1, \vec{p}(\cdot)}(G)$ (respectively, in $\left.W_{\infty, l o c}^{1, \vec{p}(\cdot)}(G)\right)$.

For the notion and basic results on quasi-minimizers in $W_{l o c}^{1, p}(\Omega)$ and in $W_{l o c}^{1, p(\cdot)}(\Omega)$ we refer to $[22-24]$ and $[17]$.

\section{Proof of Theorem 1.1}

In this section, we will prove Theorem 1.1 by using the idea and the results of Fusco and Sbordone [21]. In [21] the local boundedness of local minimizers (or local quasi-minimizers) of integral functionals with $\vec{p}$ growth is proved.

Let $f$ be as in Theorem 1.1 and $u \in W_{l o c}^{1, \vec{p}(\cdot)}(\Omega)$ be a local quasi-minimizer of $J$ over $\Omega$ in $W_{l o c}^{1, \vec{p}(\cdot)}(\Omega)$. We will prove that $u \in L_{l o c}^{\infty}(\Omega)$.

By Theorem 2.1 and the assumptions of Theorem 1.1, we can see that $J(v, E) \in \mathbb{R}$ for every $v \in W_{l o c}^{1, \vec{p}(\cdot)}(\Omega)$ and $E \subset \subset \Omega$.

Let any $x_{0} \in \Omega$ be given. By (1.1), (1.3), (1.4) and the continuity of $\vec{p}(\cdot)$, $q(\cdot)$ and $r(\cdot)$, there exists an open cube $K\left(x_{0}, R\right) \subset \subset \Omega$ such that $u$ is a quasiminimizer of $J$ over $K\left(x_{0}, R\right)$ in $W_{l o c}^{1, \vec{p}}\left(K\left(x_{0}, R\right)\right)$ with proportion coefficient 
$Q$ and the following conditions are satisfied:

$$
\begin{aligned}
p_{i,+}\left(K\left(x_{0}, R\right)\right) & <\overline{p_{-}\left(K\left(x_{0}, R\right)\right)} * \text { for } i=1,2, \ldots, N \\
q_{+}\left(K\left(x_{0}, R\right)\right) & <\overline{p_{-}\left(K\left(x_{0}, R\right)\right)} \\
r_{-}\left(K\left(x_{0}, R\right)\right) & >\max \left\{1, \frac{N}{\overline{p_{-}\left(K\left(x_{0}, R\right)\right)}}\right\}
\end{aligned}
$$

where

$$
\overrightarrow{p_{-}\left(K\left(x_{0}, R\right)\right)}=\left(p_{1,-}\left(K\left(x_{0}, R\right)\right), \ldots, p_{N,-}\left(K\left(x_{0}, R\right)\right)\right)
$$

and $\overrightarrow{p_{-}\left(K\left(x_{0}, R\right)\right)}$ is the harmonic average for $\overrightarrow{p_{-}\left(K\left(x_{0}, R\right)\right)}$.

For brevity, we will write $p_{i,-}, \overline{p_{-}},{\overline{p_{-}}}^{*}, q_{+}$and $r_{-}$instead of $p_{i,-}\left(K\left(x_{0}, R\right)\right), \overline{p_{-}\left(K\left(x_{0}, R\right)\right)}, \overline{p_{-}\left(K\left(x_{0}, R\right)\right)}{ }^{*}, q_{+}\left(K\left(x_{0}, R\right)\right)$ and $r_{-}\left(K\left(x_{0}, R\right)\right)$, respectively. We also assume ${\overline{p_{-}}}^{*}<\infty$, that is $\overline{p_{-}}<N$, otherwise use a sufficiently large positive number instead of ${\overline{p_{-}}}^{*}$.

Let $\sigma \in(0,1)$ and $\sigma R<s<t<R$. Let $\eta \in C_{0}^{\infty}(\Omega)$ be such that $\eta(x)=0$ for $x \in \Omega \backslash K\left(x_{0}, t\right), \eta(x)=1$ for $x \in K\left(x_{0}, s\right), \eta(x) \in[0,1]$ and $|D \eta(x)| \leq \frac{4}{t-s}$ for $x \in \Omega$. Let us fix $k \geq 1$. Set

$$
\varphi=-\eta(u-k)^{+} \quad \text { and } \quad v=u+\varphi
$$

where $u^{+}(x):=\max \{u(x), 0\}$. Then $v \in W_{l o c}^{1, \vec{p}(\cdot)}(\Omega)$. Putting

$$
A_{k, t}=\left\{x \in K\left(x_{0}, t\right): u(x) \geq k\right\},
$$

then $\left.v\right|_{\Omega \backslash A_{k, t}}=\left.u\right|_{\Omega \backslash A_{k, t}}$, and consequently,

$$
J\left(u, A_{k, t}\right) \leq Q J\left(v, A_{k, t}\right) .
$$

that is

$$
\int_{A_{k, t}} f(x, u, D u) \mathrm{d} x \leq Q \int_{A_{k, t}} f(x, v, D v) \mathrm{d} x .
$$

Without loss of generality we may assume that $c_{0}=1$ in (1.2). In what follows we will use $c$ as a generic positive constant, i.e., its value may change from line to line. Then, by (3.4) and (1.2), we have

$$
\begin{aligned}
& \int_{A_{k, t}}\left(\sum_{i=1}^{N}\left|D_{i} u\right|^{p_{i}(x)}-c_{1}|u|^{q(x)}-c_{1} g(x)\right) \mathrm{d} x \\
& \leq Q c_{2} \int_{A_{k, t}}\left(\sum_{i=1}^{N}\left|D_{i} v\right|^{p_{i}(x)}+|v|^{q(x)}+g(x)\right) \mathrm{d} x .
\end{aligned}
$$


For various terms of (3.5) we have the following estimations:

$$
\begin{aligned}
& \int_{A_{k, t}} \sum_{i=1}^{N}\left|D_{i} u\right|^{p_{i}(x)} \mathrm{d} x \geq \int_{A_{k, s}} \sum_{i=1}^{N}\left|D_{i} u\right|^{p_{i}(x)} \mathrm{d} x, \\
& \int_{A_{k, t}} \sum_{i=1}^{N}\left|D_{i} v\right|^{p_{i}(x)} \mathrm{d} x \\
& =\int_{A_{k, t}} \sum_{i=1}^{N}\left|(1-\eta) D_{i} u-D_{i} \eta(u-k)^{+}\right|^{p_{i}(x)} \mathrm{d} x \\
& \leq c \int_{A_{k, t} \backslash A_{k, s}} \sum_{i=1}^{N}\left|D_{i} u\right|^{p_{i}(x)} \mathrm{d} x+c \int_{A_{k, t}} \sum_{i=1}^{N}\left|\frac{u-k}{t-s}\right|^{p_{i}(x)} \mathrm{d} x \\
& \leq c \int_{A_{k, t} \backslash A_{k, s}} \sum_{i=1}^{N}\left|D_{i} u\right|^{p_{i}(x)} \mathrm{d} x+c \int_{A_{k, t}}\left|\frac{u-k}{t-s}\right|^{\overline{p-}^{*}} \mathrm{~d} x+c\left|A_{k, t}\right|, \\
& \int_{A_{k, t}}|u|^{q(x)} \mathrm{d} x=\int_{A_{k, t}}|(u-k)+k|^{q(x)} \mathrm{d} x \leq c \int_{A_{k, t}}|u-k|^{q(x)} \mathrm{d} x+c k^{q_{+}}\left|A_{k, t}\right| \\
& \leq c \int_{A_{k, t}}\left|\frac{u-k}{t-s}\right|^{\overline{p-}^{*}} \mathrm{~d} x+c k^{q+}\left|A_{k, t}\right| \\
& \int_{A_{k, t}}|v|^{q(x)} \mathrm{d} x \leq \int_{A_{k, t}}(2|u|)^{q(x)} \mathrm{d} x \leq c \int_{A_{k, t}}\left|\frac{u-k}{t-s}\right|^{\bar{p}_{-}^{*}} \mathrm{~d} x+c k^{q_{+}}\left|A_{k, t}\right| .
\end{aligned}
$$

Note that condition (3.3), i.e., $r_{-}>\frac{N}{\overline{p_{-}}}$, implies that there exists $\delta \in(0,1)$ such that $\frac{1}{r_{-}^{\prime}}=\frac{\overline{p_{-}}}{\overline{p_{-}}}+\delta$, where $\frac{1}{r_{-}^{\prime}}+\frac{1}{r_{-}}=1$. Then from $g \in L_{l o c}^{r(\cdot)}(\Omega)$ we have

$$
\int_{A_{k, t}} g(x) \mathrm{d} x \leq\|g\|_{L^{r}-\left(A_{k, t}\right)}\|1\|_{L^{r^{\prime}-\left(A_{k, t}\right)}} \leq c\left|A_{k, t}\right|^{\frac{\overline{\bar{p}_{-}}}{\bar{p}^{*}}+\delta} .
$$

Note that $\left|A_{k, t}\right| \leq k^{q+}\left|A_{k, t}\right|$ because $k \geq 1$. From (3.5)-(3.10) we obtain

$$
\begin{aligned}
\int_{A_{k, s}} \sum_{i=1}^{N}\left|D_{i} u\right|^{p_{i}(x)} \mathrm{d} x \leq & c \int_{A_{k, t} \backslash A_{k, s}} \sum_{i=1}^{N}\left|D_{i} u\right|^{p_{i}(x)} \mathrm{d} x+c \int_{A_{k, t}}\left|\frac{u-k}{t-s}\right|^{\overline{p-}^{*}} \mathrm{~d} x \\
& +c k^{q_{+}}\left|A_{k, t}\right|+c\left|A_{k, t}\right|^{\frac{\overline{\bar{p}}_{-}}{\bar{p}^{*}}+\delta}
\end{aligned}
$$

Adding $c \int_{A_{k, s}} \sum_{i=1}^{N}\left|D_{i} u\right|^{p_{i}(x)} \mathrm{d} x$ to (3.11), and dividing both sides of the resulted inequality by $1+c$, we obtain

$$
\begin{aligned}
& \int_{A_{k, s}} \sum_{i=1}^{N}\left|D_{i} u\right|^{p_{i}(x)} \mathrm{d} x \leq \theta \int_{A_{k, t}} \sum_{i=1}^{N}\left|D_{i} u\right|^{p_{i}(x)} \mathrm{d} x+\theta \int_{A_{k, R}}\left|\frac{u-k}{t-s}\right|^{\bar{p}_{-}^{*}} \mathrm{~d} x \\
& +\theta k^{q+}\left|A_{k, R}\right|+\theta\left|A_{k, R}\right|^{\frac{\overline{p_{-}}}{\overline{p_{-}}}+\delta},
\end{aligned}
$$

where $\theta=\frac{c}{1+c} \in(0,1)$. 
It is well known that it follows from (3.12), using Lemma 3.1 of [22, Chapter V], that

$$
\begin{gathered}
\int_{A_{k, \sigma R}} \sum_{i=1}^{N}\left|D_{i} u\right|^{p_{i}(x)} \mathrm{d} x \leq c \int_{A_{k, R}}\left|\frac{u-k}{R(1-\sigma)}\right|^{\bar{p}_{-}^{*}} \mathrm{~d} x+c k^{q_{+}}\left|A_{k, R}\right| \\
+c\left|A_{k, R}\right|^{\frac{\bar{p}_{-}}{\bar{p}_{-}}+\delta} .
\end{gathered}
$$

From (3.13) we have

$$
\begin{aligned}
& \int_{A_{k, \sigma R}} \sum_{i=1}^{N}\left|D_{i} u\right|^{p_{-}} \mathrm{d} x \\
& \leq \int_{A_{k, \sigma R}} \sum_{i=1}^{N}\left(1+\left|D_{i} u\right|^{p_{i}(x)}\right) \mathrm{d} x \\
& \quad \leq c \int_{A_{k, R}}\left|\frac{u-k}{R(1-\sigma)}\right|^{\overline{p_{-}} *} \mathrm{~d} x+c k^{q_{+}}\left|A_{k, R}\right|+c\left|A_{k, R}\right|^{\frac{\bar{p}_{-}}{\bar{p}_{-}^{*}}+\delta} .
\end{aligned}
$$

By Lemma 2.4 of [21], (3.14) implies that $u$ is bounded from above on $K\left(x_{0}, R / 2\right)$. Noting that $-u$ is a local quasi-minimizer of

$$
\widetilde{J}(u)=\int_{\Omega} \widetilde{f}(x, u(x), D u(x)) \mathrm{d} x,
$$

where $\tilde{f}(x, s, \xi)=f(x,-s,-\xi)$, we can see that $-u$ is also bounded from above on $K\left(x_{0}, R / 2\right)$, and thus $u \in L^{\infty}\left(K\left(x_{0}, R / 2\right)\right)$. This shows $u \in L_{l o c}^{\infty}(\Omega)$. Theorem 1.1 is proved.

Remark 3.1. If in Theorem 1.1 use $W_{\infty, l o c}^{1, \vec{p}(\cdot)}(\Omega)$ instead of $W_{l o c}^{1, \vec{p}(\cdot)}(\Omega)$, then the same assertion is true. The proof for the $W_{\infty, l o c}^{1, \vec{p}(\cdot)}(\Omega)$ case is same as the proof done above for $W_{l o c}^{1, \vec{p}(\cdot)}(\Omega)$ case. We only point out that, in the proof, when $u \in W_{\infty, l o c}^{1, \vec{p}(\cdot)}(\Omega), v=u-\eta(u-k)^{+} \in W_{\infty, l o c}^{1, \vec{p}(\cdot)}(\Omega)$.

\section{Regularity of weak solutions of $\overrightarrow{p(x)}$-Laplace type equations}

The quasi-minimizer is a very general notion which includes the local minimizer, the weak solution of corresponding elliptic equation and the weak solution of corresponding variational inequality as its special cases (see [17,22-24]). In this section we only give some applications of Theorem 1.1 to the regularity of weak solutions for $\vec{p}(\cdot)$-Laplace type equations.

Let $\Omega \subset \mathbb{R}^{N}$ be an open set. Consider the following elliptic equation in divergence form with $\overrightarrow{p(x)}$ growth

$$
-\operatorname{div} A(x, u, D u)=B(x, u, D u) \text { in } \Omega,
$$


where $A=\left(A_{1}, \ldots, A_{N}\right): \Omega \times \mathbb{R} \times \mathbb{R}^{N} \rightarrow \mathbb{R}^{N}$ and $B: \Omega \times \mathbb{R} \times \mathbb{R}^{N} \rightarrow \mathbb{R}$ satisfy the following conditions: for $(x, s, \xi) \in \Omega \times \mathbb{R} \times \mathbb{R}^{N}$,

$$
\begin{aligned}
& A(x, s, \xi) \xi \geq c_{0} \sum_{i=1}^{N}\left|\xi_{i}\right|^{p_{i}(x)}-c_{1}|s|^{q(x)}-c_{1} h(x), \\
& \left|A_{i}(x, s, \xi)\right| \leq c_{2}\left(\left|\xi_{i}\right|^{p_{i}(x)-1}+|s|^{\sigma_{i}(x)}+\mathrm{d}_{i}(x)\right), i=1,2, \ldots, N, \\
& |B(x, s, \xi)| \leq c_{3}\left(\sum_{i=1}^{N}\left|\xi_{i}\right|^{\tau_{i}(x)}+|s|^{\delta(x)}+e(x)\right)
\end{aligned}
$$

where $\vec{p}(\cdot) \in\left(C_{+}^{0}(\bar{\Omega})\right)^{N}$ satisfies $(1.1), q \in C_{+}^{0}(\bar{\Omega})$ satisfies $(1.3)$,

$$
\begin{gathered}
\sigma_{i}(x)=\frac{q(x)}{p_{i}^{\prime}(x)}, \quad \tau_{i}(x)=\frac{p_{i}(x)}{q^{\prime}(x)}, \quad \delta(x)=q(x)-1, \quad \forall x \in \Omega, i=1,2, \ldots, N, \\
h \in L_{l o c}^{1}(\Omega), \quad \mathrm{d}_{i} \in L_{l o c}^{p_{i}^{\prime}(\cdot)}(\Omega), \quad e \in L_{l o c}^{q^{\prime}(\cdot)}(\Omega), \quad h, \mathrm{~d}_{i}, e \geq 0, \quad
\end{gathered}
$$
where $\frac{1}{q^{\prime}(x)}+\frac{1}{q(x)}=1$.

Definition 4.1. $u \in W_{l o c}^{1, \vec{p}(\cdot)}(\Omega)$ (respectively, $u \in W_{\infty, l o c}^{1, \vec{p}(\cdot)}(\Omega)$ ) is called a weak solution of equation (4.1) in $W_{l o c}^{1, \vec{p}(\cdot)}(\Omega)$ (respectively, in $W_{\infty, l o c}^{1, \vec{p}(\cdot)}(\Omega)$ ) if for every $\varphi \in W_{l o c}^{1, \vec{p}(\cdot)}(\Omega)$ (respectively, $\varphi \in W_{\infty, l o c}^{1, \vec{p}(\cdot)}(\Omega)$ ) with $\operatorname{supp} \varphi \subset \subset \Omega$, there holds

$$
\int_{\Omega} A(x, u, D u) D \varphi \mathrm{d} x=\int_{\Omega} B(x, u, D u) \varphi \mathrm{d} x .
$$

Theorem 4.1. Let (1.1), (1.3), (4.2)-(4.6) hold. Suppose that $u \in W_{l o c}^{1, \vec{p}(\cdot)}(\Omega)$ (respectively, $\left.u \in W_{\infty, l o c}^{1, \vec{p}(\cdot)}(\Omega)\right)$ is a weak solution of $(4.1)$ in $W_{l o c}^{1, \vec{p}(\cdot)}(\Omega)$ (respectively, in $\left.W_{\infty, l o c}^{1, \vec{p}(\cdot)}(\Omega)\right)$. Then $u$ is a local quasi-minimizer of $J_{0}$ over $\Omega$ in $W_{\text {loc }}^{1, \vec{p}(\cdot)}(\Omega)$ (respectively, in $W_{\infty, l o c}^{1, \vec{p}(\cdot)}(\Omega)$ ), where

$$
\begin{aligned}
J_{0}(u, G) & =\int_{G}\left(\sum_{i=1}^{N}\left|D_{i} u\right|^{p_{i}(x)}+|u|^{q(x)}+g(x)\right) d x \text { for } G \subset \subset \Omega, \\
g(x) & =h(x)+\sum_{i=1}^{N}\left(d_{i}(x)\right)^{p_{i}^{\prime}(x)}+(e(x))^{q^{\prime}(x)}+1 .
\end{aligned}
$$

Proof. We only give the proof for the $W_{l o c}^{1, \vec{p}(\cdot)}(\Omega)$ case because the proof for the $W_{\infty, l o c}^{1, \vec{p}(\cdot)}(\Omega)$ case is completely similar. Now let $u \in W_{l o c}^{1, \vec{p}(\cdot)}(\Omega)$ be a weak solution of (4.1) in $W_{l o c}^{1, \vec{p}(\cdot)}(\Omega)$. Let any $x_{0} \in \Omega$ be given. Then there exists an open cube $K\left(x_{0}, R\right) \subset \subset \Omega$ such that

$$
p_{i,+}\left(K\left(x_{0}, R\right)\right)<{\overline{p_{-}\left(K\left(x_{0}, R\right)\right)}}^{*} \quad \text { for } i=1,2, \ldots, N,
$$

and

$$
q_{+}\left(K\left(x_{0}, R\right)\right)<{\overline{p_{-}\left(K\left(x_{0}, R\right)\right)}}^{*} .
$$


Let $v \in W_{l o c}^{1, \vec{p}(\cdot)}(\Omega)$ with $E:=\operatorname{supp}(u-v) \subset \subset K\left(x_{0}, R\right)$. To prove Theorem 4.1 it is sufficient to show that

$$
J_{0}(u, E) \leq Q J_{0}(v, E)
$$

where $Q \geq 1$ is a positive constant independent of $E$ and $v$.

Note that, by Theorem 2.1, for $v \in W_{l o c}^{1, \vec{p}(\cdot)}(\Omega)$ and $E \subset \subset \Omega$, we have $v \in L^{q(\cdot)}(E)$.

Putting $\varphi=u-v$, then $\varphi \in W_{l o c}^{1, \vec{p}(\cdot)}(\Omega)$ with $\operatorname{supp} \varphi=E$, and thus (4.7) holds for $\varphi=u-v$, namely

$$
\int_{E} A(x, u, D u) D u \mathrm{~d} x=\int_{E} A(x, u, D u) D v \mathrm{~d} x+\int_{E} B(x, u, D u)(u-v) \mathrm{d} x .
$$

For brevity, we will assume that $c_{0}=1$ in $(4.2)$, and write $p_{i,-}, \overline{p_{-}},{\overline{p_{-}}}^{*}$ and $q_{+}$instead of $p_{i,-}\left(K\left(x_{0}, R\right)\right), \overline{p_{-}\left(K\left(x_{0}, R\right)\right)}, \overline{p_{-}\left(K\left(x_{0}, R\right)\right)}{ }^{*}$ and $q_{+}$ $\left(K\left(x_{0}, R\right)\right)$, respectively. By the assumptions of Theorem 4.1 and Young inequality, we have

$$
\begin{aligned}
\int_{E} A(x, u, D u) D u \mathrm{~d} x & \geq \int_{E} \sum_{i=1}^{N}\left|D_{i} u\right|^{p_{i}(x)} \mathrm{d} x-c_{1} \int_{E}|u|^{q(x)} \mathrm{d} x-c_{1} \int_{E} h(x) \mathrm{d} x, \\
\int_{E} A(x, u, D u) D v \mathrm{~d} x & \leq \int_{E} \sum_{i=1}^{N}\left(\left|A_{i}(x, u, D u)\right|\left|D_{i} v\right|\right) \mathrm{d} x \\
& \leq c_{2} \int_{E} \sum_{i=1}^{N}\left(\left(\left|D_{i} u\right|^{p_{i}(x)-1}+|u|^{\sigma_{i}(x)}+\mathrm{d}_{i}(x)\right)\left|D_{i} v\right|\right) \mathrm{d} x, \\
\int_{E} B(x, u, D u)(u-v) \mathrm{d} x & \leq c_{3} \int_{E}\left(\sum_{i=1}^{N}\left|D_{i} u\right|^{\tau_{i}(x)}+|u|^{\delta(x)}+e(x)\right)|u-v| \mathrm{d} x, \\
\sum_{i=1}^{N}\left(\left|D_{i} u\right|^{p_{i}(x)-1}\left|D_{i} v\right|\right) \mathrm{d} x & \leq \varepsilon \int_{E} \sum_{i=1}^{N}\left|D_{i} u\right|^{p_{i}(x)} \mathrm{d} x+c(\varepsilon) \int_{E} \sum_{i=1}^{N}\left|D_{i} v\right|^{p_{i}(x)} \mathrm{d} x, \\
\int_{E} \sum_{i=1}^{N}\left(|u|^{\sigma_{i}(x)}\left|D_{i} v\right|\right) \mathrm{d} x & \leq c \int_{E}|u|^{q(x)} \mathrm{d} x+c \int_{E} \sum_{i=1}^{N}\left|D_{i} v\right|^{p_{i}(x)} \mathrm{d} x, \\
\int_{E} \sum_{i=1}^{N}\left(\mathrm{~d}_{i}(x)\left|D_{i} v\right|\right) \mathrm{d} x & \leq c \int_{E} \sum_{i=1}^{N}\left(\mathrm{~d}_{i}(x)\right)^{p_{i}^{\prime}(x)} \mathrm{d} x+c \int_{E} \sum_{i=1}^{N}\left|D_{i} v\right|^{p_{i}(x)} \mathrm{d} x, \\
\int_{E} \sum_{i=1}^{N}\left|D_{i} u\right|^{\tau_{i}(x)}|u-v| \mathrm{d} x & \leq \varepsilon \int_{E} \sum_{i=1}^{N}\left|D_{i} u\right|^{p_{i}(x)} \mathrm{d} x+c \int_{E}|u-v|^{q(x)} \mathrm{d} x, \\
\int_{E}|u|^{\delta(x)}|u-v| \mathrm{d} x & \leq c \int_{E}|u|^{q(x)} \mathrm{d} x+c \int_{E}|u-v|^{q(x)} \mathrm{d} x, \\
\int_{E} e(x)|u-v| \mathrm{d} x & \leq c \int_{E}(e(x))^{q^{\prime}(x)} \mathrm{d} x+c \int_{E}|u-v|^{q(x)} \mathrm{d} x, \\
\int^{q(x)} \mathrm{d} x & \leq c \int_{E}|v|^{q(x)} \mathrm{d} x+c \int_{E}|u-v|^{q(x)} \mathrm{d} x .
\end{aligned}
$$


From (4.11) and the above inequalities, choosing $\varepsilon>0$ small enough, we can obtain that

$$
\begin{aligned}
& \int_{E} \sum_{i=1}^{N}\left|D_{i} u\right|^{p_{i}(x)} \mathrm{d} x \leq c \int_{E}|u-v|^{q(x)} \mathrm{d} x+c \int_{E}\left(\sum_{i=1}^{N}\left|D_{i} v\right|^{p_{i}(x)}+|v|^{q(x)}\right) \mathrm{d} x \\
& \quad+c \int_{E}\left(h(x)+\sum_{i=1}^{N}\left(\mathrm{~d}_{i}(x)\right)^{p_{i}^{\prime}(x)}+(e(x))^{q^{\prime}(x)}\right) \mathrm{d} x .
\end{aligned}
$$

We may assume that

$$
\int_{E} \sum_{i=1}^{N}\left|D_{i} u\right|^{p_{i}(x)} \mathrm{d} x+\int_{E}|u|^{q(x)} \mathrm{d} x>\int_{E} \sum_{i=1}^{N}\left|D_{i} v\right|^{p_{i}(x)} \mathrm{d} x
$$

because otherwise inequality (4.10) already holds. We may also assume that the cube $K\left(x_{0}, R\right)$ is already taken sufficiently small such that

$$
\int_{K\left(x_{0}, R\right)} \sum_{i=1}^{N}\left|D_{i} u\right|^{p_{i}(x)} \mathrm{d} x+\int_{K\left(x_{0}, R\right)}\left(|u|^{q(x)}+1\right) \mathrm{d} x<1 .
$$

For $\int_{E}|u-v|^{q(x)} \mathrm{d} x$ we have

$$
\int_{E}|u-v|^{q(x)} \mathrm{d} x \leq \varepsilon \int_{E}|u-v|^{\bar{p}_{-}^{*}} \mathrm{~d} x+c(\varepsilon) \int_{E} 1 \mathrm{~d} x .
$$

By Proposition 2.1, (4.14) and (4.13), we have

$$
\begin{aligned}
& \int_{E}|u-v|^{\overline{p-}^{*}} \mathrm{~d} x \\
& \leq c\left(\sum_{i=1}^{N}\left(\int_{E}\left|D_{i} u-D_{i} v\right|^{p_{i,-}} \mathrm{d} x\right)^{\frac{1}{p_{i,-}}}\right)^{{\overline{p_{-}}}^{*}} \\
& \leq c \sum_{i=1}^{N}\left(\int_{E}\left(\left|D_{i} u\right|^{p_{i,-}}+\left|D_{i} v\right|^{p_{i,-}}\right) \mathrm{d} x\right)^{\frac{\bar{p}_{-}^{*}}{p_{i,-}}} \\
& \leq c \sum_{i=1}^{N}\left(\int_{E}\left(\left|D_{i} u\right|^{p_{i}(x)}+\left|D_{i} v\right|^{p_{i}(x)}+2\right) \mathrm{d} x\right)^{\frac{\overline{p-}_{i,-}^{*}}{p_{i,-}}} \\
& \leq c \sum_{i=1}^{N}\left(\left(\int_{E}\left|D_{i} u\right|^{p_{i}(x)} \mathrm{d} x\right)^{\frac{\overline{p_{-}} *}{p_{i,-}}}+\left(\int_{E}\left|D_{i} v\right|^{p_{i}(x)} \mathrm{d} x\right)^{\frac{p_{-} *}{p_{i,-}}}+\left(\int_{E} 1 \mathrm{~d} x\right)^{\frac{\overline{p_{-}} *}{p_{i,-}}}\right) \\
& \leq c \sum_{i=1}^{N}\left(\left(\int_{E}\left|D_{i} u\right|^{p_{i}(x)} \mathrm{d} x\right)+\left(\int_{E}\left|D_{i} v\right|^{p_{i}(x)} \mathrm{d} x\right)+\left(\int_{E} 1 \mathrm{~d} x\right)\right) \\
& \leq c \sum_{i=1}^{N} \int_{E}\left|D_{i} u\right|^{p_{i}(x)} \mathrm{d} x+c \int_{E}|u|^{q(x)} \mathrm{d} x+c \int_{E} 1 \mathrm{~d} x .
\end{aligned}
$$


By (4.12), (4.15) and (4.16), choosing $\varepsilon>0$ in (4.15) small enough, we can obtain that

$$
\begin{aligned}
& \int_{E}\left(\sum_{i=1}^{N}\left|D_{i} u\right|^{p_{i}(x)}+|u|^{q(x)}+g(x)\right) \mathrm{d} x \\
& \leq Q \int_{E}\left(\sum_{i=1}^{N}\left|D_{i} v\right|^{p_{i}(x)}+|v|^{q(x)}+g(x)\right) \mathrm{d} x,
\end{aligned}
$$

where $g$ is as in (4.9), and $Q \geq 1$ is a positive constant independent of $G$ and $v$. This shows that (4.10) holds.

From Theorems 4.1 and 1.1 we immediately obtain the following

Theorem 4.2. Let (1.1), (1.3), (4.2)-(4.6) hold. Let $g$ be as in (4.9) and g satisfy assumption (1.4). Suppose that $u \in W_{\text {loc }}^{1, \vec{p}(\cdot)}(\Omega)$ (respectively, $u \in W_{\infty, l o c}^{1, \vec{p}(\cdot)}(\Omega)$ ) is a weak solution of $(4.1)$ in $W_{\text {loc }}^{1, \vec{p}(\cdot)}(\Omega)$ (respectively, in $W_{\infty, l o c}^{1, \vec{p}(\cdot)}(\Omega)$ ). Then $u \in L_{l o c}^{\infty}(\Omega)$.

Lieberman [31] studied a very wide class of anisotropic elliptic equations of the form (4.1) and proved that, under very general assumptions, any locally bounded weak solution of such an equation must have a locally bounded gradient. Combining Theorem 4.2 and the result of [31] we can obtain that, under the assumptions of Theorem 4.2, if in addition, $A$ and $B$ satisfy the conditions required in [31], then for every weak solution $u$ of $(4.1),|D u| \in L_{\text {loc }}^{\infty}(\Omega)$.

Now let us consider the following Dirichlet boundary value problem

$$
\left\{\begin{array}{c}
-\operatorname{div} A(x, u, D u)=B(x, u, D u) \text { in } \Omega \\
u=0 \text { on } \partial \Omega
\end{array},\right.
$$

where $\Omega \subset \mathbb{R}^{N}$ is a bounded domain, $A$ and $B$ are as in Theorem 4.1.

Definition 4.2. $u \in W_{0}^{1,1}(\Omega) \cap W^{1, \vec{p}(\cdot)}(\Omega)$ (respectively, $u \in W_{0}^{1, \vec{p}(\cdot)}(\Omega)$ ) is called a weak solution of problem $(4.18)$ in $W_{0}^{1,1}(\Omega) \cap W^{1, \vec{p}(\cdot)}(\Omega)$ (respectively, in $W_{0}^{1, \vec{p}(\cdot)}(\Omega)$ ) if for every $\varphi \in W_{0}^{1,1}(\Omega) \cap W^{1, \vec{p}(\cdot)}(\Omega)$ (respectively, $\left.\varphi \in W_{0}^{1, \vec{p}(\cdot)}(\Omega)\right)$, (4.7) holds.

Theorem 4.3. Let $\Omega \subset \mathbb{R}^{N}$ be a bounded domain with Lipschitz boundary. Let $A$ and $B$ satisfy conditions (4.2)-(4.4), and let (1.1), (1.3) and (4.5) hold for for $x \in \bar{\Omega}$ (not only for $x \in \Omega$ ), and

$$
h \in L^{1}(\Omega), d_{i} \in L^{p_{i}^{\prime}(\cdot)}(\Omega), e \in L^{q^{\prime}(\cdot)}(\Omega), h, d_{i}, e \geq 0 .
$$

Let $g$ be as in (4.9), $g \in L^{r(\cdot)}(\Omega)$ and (1.4) hold for $x \in \bar{\Omega}$. Suppose that $u \in W_{0}^{1,1}(\Omega) \cap W^{1, \vec{p}(\cdot)}(\Omega)$ (respectively, $u \in W_{0}^{1, \vec{p}(\cdot)}(\Omega)$ ) is a weak solution of problem $(4.18)$ in $W_{0}^{1,1}(\Omega) \cap W^{1, \vec{p}(\cdot)}(\Omega)$ (respectively, in $W_{0}^{1, \vec{p}(\cdot)}(\Omega)$ ). Then $u \in L^{\infty}(\Omega)$.

Proof. Let any $x_{0} \in \bar{\Omega}$ be given. In the case when $x_{0} \in \Omega$, applying Theorem 4.2 immediately, we know that there exists a neighborhood $\Omega_{x_{0}} \subset \Omega$ of $x_{0}$ such that $u \in L^{\infty}\left(\Omega_{x_{0}}\right)$. In the case when $x_{0} \in \partial \Omega$, by Definition 4.2 and 
the assumptions of Theorem 4.3, using arguments similar to those done in the proof of Theorems 4.1 and 1.1, we can prove that there exists an open cube $K\left(x_{0}, R\right)$ with sufficiently small $R$ such that $u \in L^{\infty}\left(K\left(x_{0}, R / 2\right) \cap \bar{\Omega}\right)$. Thus, by the compactness of $\bar{\Omega}$, we can obtain that $u \in L^{\infty}(\Omega)$.

Note that in Theorems 1.1, 2.1, 4.1, 4.2 and 4.3, we suppose that $p_{i}$ is continuous but do not suppose that $p_{i}$ is log-Hölder continuous.

Let us consider the following Dirichlet problem, a special case of (4.18),

$$
\left\{\begin{array}{c}
-\sum_{i=1}^{N} D_{i}\left(\left(r_{i}^{2}+\left|D_{i} u\right|^{2}\right)^{\frac{p_{i}(x)-2}{2}} D_{i} u\right)=g(x, u) \text { in } \Omega \\
u=0 \text { on } \partial \Omega
\end{array} .\right.
$$

The equation in (4.19) is a special case of the equations considered by Lieberman [31] (see Example 8 of [31]). Combining Theorem 4.3 and the result of Lieberman [31], we give the following Theorem 4.4. Note that, in Theorem 4.4, since it is supposed that $p_{i}: \bar{\Omega} \rightarrow \mathbb{R}$ is Lipschitz continuous, so by Theorem $2.2, W_{0}^{1, \vec{p}(\cdot)}(\Omega)=W_{0}^{1,1}(\Omega) \cap W^{1, \vec{p}(\cdot)}(\Omega)$.

Theorem 4.4. Let $\Omega \subset \mathbb{R}^{N}$ be a bounded domain with Lipschitz boundary. Let $r_{i} \geq 0$ and $p_{i}: \bar{\Omega} \rightarrow \mathbb{R}$ be Lipschitz continuous for $i=1,2, \ldots, N$, and (1.1) hold for all $x \in \bar{\Omega}$. Let $g: \bar{\Omega} \times \mathbb{R} \rightarrow \mathbb{R}$ be a Carathéodory function satisfying the condition

$$
|g(x, t)| \leq c+c|t|^{q(x)-1} \text { for } \quad x \in \Omega \text { and } t \in \mathbb{R},
$$

where $c$ is a positive constant, $q \in C_{+}^{0}(\bar{\Omega})$ satisfies (1.3) for $x \in \bar{\Omega}$. Suppose that $u \in W_{0}^{1, \vec{p}(\cdot)}(\Omega)$ is a weak solution of problem (4.19). Then $|D u| \in L^{\infty}(\Omega)$ and thus $u$ is Lipschitz continuous on $\bar{\Omega}$.

Proof. Let $\left.u \in W_{0}^{1, \vec{p}(\cdot)}(\Omega)\right)$ is a weak solution of problem (4.19). By Theorem 4.3, $u \in L^{\infty}(\Omega)$. Setting $h(x)=g(x, u(x))$, then $h \in L^{\infty}(\Omega)$ and $u$ is a bounded weak solution of the problem

$$
\left\{\begin{array}{c}
-\sum_{i=1}^{N} D_{i}\left(\left(r_{i}^{2}+\left|D_{i} u\right|^{2}\right)^{\frac{p_{i}(x)-2}{2}} D_{i} u\right)=h(x) \text { in } \Omega \\
u=0 \text { on } \partial \Omega
\end{array}\right.
$$

Applying immediately the result of $[31$, Example 8] to (4.20), we have $|D u| \in$ $L_{\text {loc }}^{\infty}(\Omega)$. For each $x_{0} \in \partial \Omega$, using arguments similar to those done in [31] for the case that $x_{0} \in \Omega$, we can obtain that there exists an open neighborhood $\Omega_{x_{0}}$ of $x_{0}$ such that $|D u| \in L^{\infty}\left(\Omega_{x_{0}} \cap \Omega\right)$. Thus, by the compactness of $\bar{\Omega}$, $|D u| \in L^{\infty}(\Omega)$.

Note that in Theorem 4.4, by the explanation given in [31, Example 8], it is permissible that $r_{i}=0$. In the non-degenerate case, that is when $r_{i}>0$ for all $i=1,2, \ldots, N$, in a standard way, it is easy to obtain the further regularity of weak solutions of (4.19) provided $\vec{p}(\cdot), g$ and $\partial \Omega$ are smooth enough (see e.g. [34]). It is well known that the $C^{1, \alpha}$-regularity of weak solutions of the $p(x)$-Laplacian equations is obtained under the assumption that $p(\cdot)$ is Hölder 
continuous (see $[2,14]$ ). The $C^{1, \alpha}$-regularity of weak solutions of (4.19) with $r_{i}=0$ under suitable assumptions is an interesting research topic.

Similarly to the Dirichlet boundary value problem (4.18), we can also consider the Neumann boundary value problem

$$
\left\{\begin{array}{c}
-\operatorname{div} A(x, u, D u)=B(x, u, D u) \text { in } \Omega \\
\frac{\partial u}{\partial \nu}=0 \text { on } \partial \Omega
\end{array},\right.
$$

where $\Omega \subset \mathbb{R}^{N}$ is a rectangular domain, $\nu$ is the outward unit normal to $\partial \Omega$, $A$ and $B$ are as in Theorem 4.3.

Definition 4.3. $u \in W^{1, \vec{p}(\cdot)}(\Omega)$ (respectively, $u \in W_{\infty}^{1, \vec{p}(\cdot)}(\Omega)$ ) is called a weak solution of problem $(4.21)$ in $W^{1, \vec{p}(\cdot)}(\Omega)$ (respectively, in $W_{\infty}^{1, \vec{p}(\cdot)}(\Omega)$ ) if for every $\varphi \in W^{1, \vec{p}(\cdot)}(\Omega)$ (respectively, $\varphi \in W_{\infty}^{1, \vec{p}(\cdot)}(\Omega)$ ), (4.7) holds.

Similarly to Theorem 4.3, we have

Theorem 4.5. Let $\Omega \subset \mathbb{R}^{N}$ be a rectangular domain, and let $A$ and $B$ are as in Theorem 4.3. Suppose that $u \in W^{1, \vec{p}(\cdot)}(\Omega)$ (respectively, $u \in W_{\infty}^{1, \vec{p}(\cdot)}(\Omega)$ ) is a weak solution of problem $(4.21)$ in $W^{1, \vec{p}(\cdot)}(\Omega)$ (respectively, in $W_{\infty}^{1, \vec{p}(\cdot)}(\Omega)$ ). Then $u \in L^{\infty}(\Omega)$.

Similarly to problem (4.19), let us consider the following Neumann boundary value problem, a special case of (4.21):

$$
\left\{\begin{array}{c}
-\sum_{i=1}^{N} D_{i}\left(\left(r_{i}^{2}+\left|D_{i} u\right|^{2}\right)^{\frac{p_{i}(x)-2}{2}} D_{i} u\right)=g(x, u) \text { in } \Omega \\
\frac{\partial u}{\partial \nu}=0 \text { on } \partial \Omega
\end{array} .\right.
$$

Similarly to Theorem 4.4, we have

Theorem 4.6. Let $\Omega \subset \mathbb{R}^{N}$ be a rectangular domain. Let $r_{i}, p_{i}$ and $g$ be as in Theorem 4.4. Suppose that $\left.u \in W^{1, \vec{p}(\cdot)}(\Omega)\right)$ is a weak solution of problem (4.22). Then $u$ is Lipschitz continuous on $\bar{\Omega}$.

\section{Acknowledgements}

The author is grateful to the referee for valuable comments and suggestions.

\section{References}

[1] Acerbi, E., Fusco, N.: Partial regularity under anisotropic $(p, q)$ growth conditions. J. Differ. Equ. 107, 46-67 (1994)

[2] Acerbi, E., Mingione, G.: Regularity results for a class of functionals with nonstandard growth. Arch. Rat. Mech. Anal. 156, 121-140 (2001)

[3] Antontsev, S., Shmarev, S.: Elliptic equations with anisotropic nonlinearity and nonstandard conditions. In: Chipot, M., Quittner, P., Elsevier B.V. (eds) Handbook of Differential Equations, Stationary Partial Differential Equations, vol. 3, North Holland, Amsterdam, pp. 1-100 (2006) 
[4] Bhattacharya, T., Leonetti, F.: On improved regularity of weak solutions of some degenerate, anisotropic elliptic systems. Ann. Mat. Pure Appl. 170, 241$255(1996)$

[5] Bildhauer, M., Fuchs, M.: $C^{1, \alpha}$-solutions to non-autonomous anisotropic variational problems. Calc. Var. 24, 309-340 (2005)

[6] Cianchi, A.: Local boundedness of minimizers of anisotropic functionals. Ann. Inst. H. Poincaré Anal. Non linéaire 17, 147-168 (2000)

[7] Cupini, G., Marcellini, P., Mascolo, E.: Regularity under sharp anisotropic general growth conditions. Discret. Continuous Dyn. Syst. B 11, 66-86 (2009)

[8] Cupini, G., Guidorzi, M., Mascolo, E.: Regularity of minimizers of vectorial integrals with $p-q$ growth. Nonlinear Anal. 54, 591-616 (2003)

[9] Diening, L.: Maximal function on generalized Lebesgue spaces $L^{p(\cdot)}$. Math. Inequal. Appl. 7, 245-253 (2004)

[10] Diening, L., Ettwein, F., Růžička, M.: $C^{1, \alpha}$-regularity for electrorheological fluids in two dimensions. Nonlinear Differ. Equ. Appl. 14, 207-217 (2007)

[11] Edmunds, D.E., Rákosník, J.: Sobolev embeddings with variable exponent. Studia Math. 143, 267-293 (2000)

[12] Esposito, L., Leonetti, F., Mingione, G.: Sharp regularity for functionals with $(p, q)$ growth. J. Diff. Equ. 204, 5-55 (2004)

[13] Fan, X.: The regularity of Lagrangians $f(x, \xi)=|\xi|^{\alpha(x)}$ with Hölder exponents $\alpha(x)$. Acta Math. Sinica (N.S.) 12, 254-261 (1996)

[14] Fan, X.: Global $C^{1, \alpha}$ regularity for variable exponent elliptic equations in divergence form. J. Differ. Equ. 235, 397-417 (2007)

[15] Fan, X., Zhao, D.: Regularity of minimizers of variational integrals with continuous $p(x)$-growth conditions. Chin. J. Contemp. Math. 17, 327-336 (1996)

[16] Fan, X., Zhao, D.: A class of De Giorgi type and Hölder continuity. Nonlinear Anal. 36, 295-318 (1999)

[17] Fan, X., Zhao, D.: The quasi-minimizer of integral functionals with $m(x)$ growth conditions. Nonlinear Anal. 39, 807-816 (2000)

[18] Fan, X., Zhao, D.: On the spaces $L^{p(x)}(\Omega)$ and $W^{m, p(x)}(\Omega)$. J. Math. Anal. Appl. 263, 424-446 (2001)

[19] Fragalà, I., Gazzola, F., Kawohl, B.: Existence and nonexistence results for anisotropic quasilinear elliptic equations. Ann. I.H. Poincaré Anal. Non linéaire 21, 715-734 (2004)

[20] Fragalà, I., Gazzola, F., Lieberman, G.: Regularity and nonexistence results for anisotropic quasilinear elliptic equations in convex domains. Discrete and Continuous Dynamical Systems, Supplement vol., pp. 280-286 (2005) 
[21] Fusco, N., Sbordone, C.: Some remarks on the regularity of minima of anisotropic integrals. Commun. Partial Differ. Equ. 18, 153-167 (1993)

[22] Giaquinta, M.: Multiple integrals in the calculus of variations and nonlinear elliptic systems. Princeton University Press, Princeton (1983)

[23] Giaquinta, M., Giusti, E.: Quasi-minima. Ann. Inst. H. Poincaré Anal. Non linéaire 1, 79-107 (1984)

[24] Giusti, E.: Direct methods in the calculus of variations. World Scientific, Singapore (2003)

[25] Habermann, J., Zatorska-Goldstein, A.: Regularity for minimizers of functionals with nonstandard growth by $\mathcal{A}$-harmonic approximation. Nonlinear Differ. Equ. Appl. 15, 169-194 (2008)

[26] Harjulehto, P., Hästö, P., Lê, U.V., Nuortio, M.: Overview of differential equations with non-standard growth (Preprint)

[27] Ji, C.: An eigenvalue of an anisotropic quasilinear elliptic equation with vatiable exponent and Neumann boundary condition. Nonlinear Anal. 71, 45074514 (2009)

[28] Kováčik, O., Rákosník, J.: On spaces $L^{p(x)}$ and $W^{k, p(x)}$. Czechoslo. Math. J. 41(116), 592-618 (1991)

[29] Kruzhkov, S.N., Kolodii, I.M.: On the theory of embedding of anisotropic Sobolev spaces. Russ. Math. Surv. 38, 188-189 (1983)

[30] Li, J.-J.: Local behaviour of solutions of anisotropic elliptic equations. Nonlinear Anal. 35, 617-628 (1999)

[31] Lieberman, G.M.: Gradient estimates for anisotropic elliptic equations. Adv. Differ. Equ. 10, 767-812 (2005)

[32] Liskevich, V., Skrypnik, I.I.: Hölder continuity of solutions to an anisotropic elliptic equation. Nonlinear Anal. 71, 1699-1708 (2009)

[33] Marcellini, P.: Regularity of minimizers of integrals of the calculus of variations with non standard growth conditions. Arch. Rat. Mech. Anal. 105, 267-284 (1989)

[34] Marcellini, P.: Regularity and existence of solutions of elliptic equations with $p, q$-growth conditions. J. Differ. Equ. 90, 1-30 (1991)

[35] Mihăilescu, M., Moroşanu, G.: Existence and multiplicity of solutions for an anisotropic elliptic problem involving variable exponent growth conditions. Appl. Anal. (in Press)

[36] Mihăilescu, M., Pucci, P., Rădulescu, V.: Nonhomogeneous boundary value problems in anisotropic Sobolev spaces. C. R. Acad. Sci. Paris Ser. I 345, 561-566 (2007) 
[37] Mihăilescu, M., Pucci, P., Rădulescu, V.: Eigenvalue problems for anisotropic quasilinear elliptic equations with variable exponent. J. Math. Anal. Appl. 340, 687-698 (2008)

[38] Rákosník, J.: Some remarks to anisotropic Sobolev spaces I. Beiträge Zur Anal. 13, 55-68 (1979)

[39] Rákosník, J.: Some remarks to anisotropic Sobolev spaces II. Beiträge Zur Anal. 15, 127-140 (1981)

[40] Růžička, M.: Electrorheological fluids: modeling and mathematical theory. Springer, Berlin (2000)

[41] Samko, S.: Denseness of $C_{0}^{\infty}\left(\mathbb{R}^{N}\right)$ in the generalized Sobolev spaces $W^{m, p(x)}\left(\mathbb{R}^{N}\right)$. Dokl. Akad. Nauk SSSR 369, 451-454 (1999)

[42] Troisi, M.: Teoremi di inclusione per spazi di Sobolev non isotropi. Ricerche Mat. 18, 3-24 (1969)

[43] Troisi, M.: Ulteriori contributi alla teoria degli spazi di Sobolev non isotropi. Ricerche Math. 20, 90-117 (1971)

[44] Zhikov, V.V.: On Lavrentiev's phenomen. Russ. J. Math. Phys. 3, 249-269 (1995)

[45] Zhikov, V.V.: Meyer-type estimates for solving the nonlinear Stokes system. Differ. Equ. 33, 108-115 (1997)

X. Fan

Department of Mathematics,

Lanzhou University,

Lanzhou 730000, China

e-mail: fanxl@lzu.edu.cn

Received: 12 October 2009.

Accepted: 12 March 2010. 